\title{
High Pressure Studies of Potassium Perchlorate.
}

\author{
Michael Pravica $^{1 *}$, Yonggang Wang ${ }^{1,2}$, Daniel Sneed ${ }^{1}$, Sharissa Reiser ${ }^{1}$, Melanie White ${ }^{1}$ \\ ${ }^{1}$ High Pressure Science and Engineering Center (HiPSEC) and Department of Phys- \\ ics, University of Nevada Las Vegas (UNLV), Las Vegas, Nevada, 89154-4002, Unit- \\ ed States \\ ${ }^{2}$ High Pressure Synergetic Consortium, Geophysical Laboratory, Carnegie Institu- \\ tion of Washington, Argonne, Illinois 60439, United States
}

Two experiments are reported on $\mathrm{KClO}_{4}$ at extreme conditions. A static high pressure Raman study was first conducted to $18.9 \mathrm{GPa}$. Evidence for at least two new phases was observed: one between 2.4 and 7.7 GPa (possibly sluggish), and the second near 11.7 GPa. Then, the x-ray induced decomposition rate of potassium perchlorate $\left(\mathrm{KClO}_{4} \stackrel{h v}{\longrightarrow} \mathrm{KCl}+2 \mathrm{O}_{2}\right)$ was studied up to $15.2 \mathrm{GPa}$. The timedependent growth of $\mathrm{KCl}$ and $\mathrm{O}_{2}$ was monitored. The decomposition rate slowed at higher pressures. We present the first direct evidence for $\mathrm{O}_{2}$ crystallization at higher pressures, demonstrating that $\mathrm{O}_{2}$ molecules segregate at high pressure.

*Corresponding author e-mail: pravica@physics.unlv.edu 


\section{Introduction:}

One critical factor determining the rate and progress of chemical reactions is the ability to rapidly and efficiently deliver mobile oxidants/reactants such as molecular oxygen. Related to this, an outstanding challenge in chemistry pertains to better understanding the rapid production and diffusion of oxygen during detonation conditions inside isolated chambers/shells. During detonation, a shockwave is generated which impresses very high pressures (of the order of $10 \mathrm{GPa}$ ) and high temperatures upon reactant molecules [1]. Gaining insight into the mobility of oxygen [2] at extreme conditions of pressure and temperature, therefore, would aid explosives chemistry under extreme conditions due to the critical need for rapid delivery of oxygen as a reactant. Also, understanding how mobile reactants such as hydrogen and oxygen [3], or fluorine and oxygen mix or segregate and react [4] under extreme conditions would be very beneficial for developing novel routes of chemical synthesis.

In this spirit, we have been investigating the $\mathrm{x}$-ray induced decomposition of potassium halates $\left(\mathrm{KClO}_{3}\right.$ [2, 5-8], $\mathrm{KClO}_{4}$ [3-4, 9], $\mathrm{KBrO}_{3}$ [9], and $\mathrm{KIO}_{3}$ [8-9]) into molecular oxygen and potassium chloride using penetrating, highly focused, and highly ionizing white $[1-2,4,6]$ and monochromatic hard x-rays [7, 8] from the Advanced Photon Source (APS). For example, we have observed the following acatalytic chemical reaction when irradiating $\mathrm{KClO}_{4}$ (a powerful oxidizer):

$$
\mathrm{KClO}_{4} \stackrel{h v}{\longrightarrow} \mathrm{KCl}+2 \mathrm{O}_{2}
$$

The objective of our efforts entailed seeking decomposition reactions that can be photochemically induced to produce mobile reactants such as $\mathrm{O}_{2}$ [2-9], $\mathrm{N}_{2}[10], \mathrm{H}_{2}[3,6]$, $\mathrm{Cl}_{2}$ [11-12], and most recently $\mathrm{F}_{2}[4,13]$ inside a diamond anvil cell (DAC) and other 
sealed chambers, in situ. As we have been using $\mathrm{KClO}_{4}$ extensively in our experiments to produce molecular oxygen in situ [9], better-understanding its high pressure behavior in its own right would be highly advantageous for our efforts to develop useful hard x-ray photochemistry [2-13].

In earlier studies, we observed a strong phase-dependence in the x-ray induced decomposition rate of $\mathrm{KClO}_{3}$ at high pressure [7] which was later theoretically modeled [14]. In that theoretical study, it was ascertained that $\mathrm{KClO}_{3}$ likely decomposes into $\mathrm{KClO}_{4}$ when in the high pressure Rhombohedral phase (above 2 GPa) [14] which agreed very well with our experimental $\mathrm{x}$-ray decomposition studies [7]. The presumed reaction is [14]:

$$
4 \mathrm{KClO}_{3 \text { (rhombohedral) }} \stackrel{h v}{\longrightarrow} 3 \mathrm{KClO}_{4(\text { orthorhombic) }}+\mathrm{KCl}_{(\mathrm{B} 2)}
$$

Thus, to better understand the decomposition mechanisms associated with $\mathrm{KClO}_{3}$ that produce oxygen under extreme conditions, it is important to understand the decomposition of $\mathrm{KClO}_{4}$ under similar conditions. We were also interested in determining if there were any phase transitions associated with $\mathrm{KClO}_{4}$ which might then have phase dependent decomposition rates as well. Potassium perchlorate is an important oxidizer for a variety of chemical reactions including those associated with energetic materials [1] and thus, in some sense, our efforts would aid in better-understanding the production of oxygen in myriad oxidation reactions at high pressure including a better understanding of the performance of this compound during shock wave conditions.

Prior studies have observed that $\mathrm{KClO}_{4}$ decomposes faster than $\mathrm{KClO}_{3}$ [9] and, as such, the former compound can be useful in more rapidly delivering oxygen in situ. Earlier studies demonstrated that pressure can be a useful mechanism to slow hard x-ray in- 
duced decomposition reactions [15-16], offering the potential of chemical control. Yet, $\mathrm{KClO}_{4}$ has been little studied at high pressure and ambient temperature to the best of our knowledge. There is one high pressure study that we are aware of [17] up to $1.5 \mathrm{GPa}$ (beyond our own earlier studies [3-4, 9]) which was based on high temperature differential thermal analysis. Another study [18] used the oxidizer to synthesize a superconductor, $(\mathrm{Ca}, \mathrm{K})_{2} \mathrm{CuO}_{2} \mathrm{Cl}_{2}$, via high pressure (5 GPa) and high temperature $900{ }^{\circ} \mathrm{C}$. A third study examined the vibrational effects of $\mathrm{KMnO}_{4}$ trapped in a potassium perchlorate matrix up to 2.4 GPa [19] but again, the focus of refs. 18-19 did not entail any fundamental investigations of $\mathrm{KClO}_{4}$ by itself with pressure.

Solid $\mathrm{KClO}_{4}$ has a orthorhombic unit cell with Pnma symmetry [20-21], and with four formula units in the primitive cell and the $\mathrm{ClO}_{4}{ }^{-}$and $\mathrm{K}^{+}$ions are situated at sites with $C_{s}$ symmetry [20] at ambient conditions; $\mathrm{a}=8.866 \AA, \mathrm{b}=5.666 \AA$ and c $=7.254 \AA$ [21]. No phase transitions were observed within the $1.5 \mathrm{GPa}$ pressure range [17]. No phase transitions were observed in the $\mathrm{KMnO}_{4} / \mathrm{KClO}_{4}$ mixture up to $5.5 \mathrm{GPa}$ though this is a different experimental system and thus not directly comparable to pure $\mathrm{KClO}_{4}[19]$.

Therefore, with an aim of better-understanding the high pressure behavior of this very important oxidizer [22] including a determination of how pressure affects the hard x-ray induced decomposition rate, we conducted a static high pressure Raman experiment of $\mathrm{KClO}_{4}$ - the first ever that we are aware of with pressure - and then performed synchrotron x-ray induced decomposition experiments by fixing the x-ray irradiating energy and altering the pressure using a diamond anvil cell (DAC). 


\section{Experimental:}

In both sets of experiments, a symmetric-style DAC was employed to pressurize the samples. Stainless steel gaskets were preindented to 50 $\mu$ m initial thickness using 300 $\mu$ m culet diameter, ultralow fluorescence Raman-quality diamonds. Roughly $100 \mu \mathrm{m}$ diameter holes were drilled into the gaskets via electric discharge machining to contain the samples. The first experiment (Raman spectroscopy) was performed at the Pravica group Raman facility. A Spectra Physics ${ }^{\circledR}$ Ar ion laser tuned to $514.5 \mathrm{~nm}$ and set at 100 $\mathrm{mW}$ (20 mW at the sample) served as the excitation source. Rayleigh scattered light was removed using a Kaiser optics ${ }^{\circledR} 514.5$ nm holographic notch filter. Inelasticallyscattered Raman light was directed into a Jobin Yvon U1000® spectrometer, dispersing the scattered light, which was then detected via an ISA Instruments Spectrum One ${ }^{\circledR}$ detector. The Raman spectrometer instrumental resolution was $\sim 1 \mathrm{~cm}^{-1}$. Acquisitions typically required 60 sec to complete.

The second set of experiments was conducted at the 16 ID-B beamline of the High Pressure-Collaborative Access Team (HP-CAT) at the Advanced Photon Source (APS). Monochromatic hard x-rays (25 keV) were directed to decompose the $\mathrm{KClO}_{4}$ sample at various pressures. The $\mathrm{x}$-ray beam was $\sim 5 \mu \mathrm{m} \times 5 \mu \mathrm{m}$ in dimensions which was much smaller than the diameter of the sample hole $(\sim 100 \mu \mathrm{m})$. This enabled us to perform multiple experiments using the same sample at different virgin/unirradiated locations within the same sample [7, 15-16]. If there were no longer any well-separated and virgin/unirradiated regions (based upon a visual check of the sample under a microscope) then the gasket was emptied, cleaned, reloaded, and repressurized with fresh unirradiated $\mathrm{KClO}_{4}$ 
No pressure transmitting medium was used in these experiments to match the conditions of our prior experiments using $\mathrm{KClO}_{4}$ and $\mathrm{KClO}_{3}$.

\section{Results (Raman Experiment):}

The Raman spectra are presented as a function of pressure in Figure 1. We note in passing that there was no evidence of laser induced damage in our samples over the course of our measurements. The ambient spectrum agrees well with that of prior studies [19-20]. The line near $460 \mathrm{~cm}^{-1}$ derives from the $v_{2}$ vibration [19-20]. The modes near $640 \mathrm{~cm}^{-1}$ commence with the $v_{2}$ vibration which split in the ordered crystal [19-20]. The peak near $940 \mathrm{~cm}^{-1}$ represents the $v_{1}$ vibrational mode with $A_{1}$ symmetry [19-20]. Finally, two small peaks near 1090 and $1120 \mathrm{~cm}^{-1}$ stem from the $v_{3}$ mode [19-20].

With increase in pressure, many lines shift toward higher energies and broaden, which is typical in many high pressure studies. Near $2.4 \mathrm{GPa}$, one new peak emerges near 760 $\mathrm{cm}^{-1}$. As pressure was increased to $7.7 \mathrm{GPa}$, a new peak emerges near $1110 \mathrm{~cm}^{-1}$ (see Figs. 1 and 2) as the two peaks near 1130 and $1150 \mathrm{~cm}^{-1}$ start merging. The peak near $920 \mathrm{~cm}^{-1}$ begins to disappear.

At $11.7 \mathrm{GPa}$, a new mode emerges near $460 \mathrm{~cm}^{-1}$ (on the left shoulder of the relatively prominent mode near $490 \mathrm{~cm}^{-1}$ ). One or possibly two new peaks also begin to appear

on the left shoulder of the peak near $640 \mathrm{~cm}^{-1}$. The two modes near $1150 \mathrm{~cm}^{-1}$ merge into one broadened peak.

Above this pressure, the modes broaden significantly and reduce in intensity with the exception of the higher frequency mode $\left(v_{1}\right)$ that is now shifted to near $1000 \mathrm{~cm}^{-1}$ and which maintains most of its intensity despite the broadening. The $v_{1}$ mode is independent 
of the orientation of the $\mathrm{ClO}_{4}{ }_{4}^{-}$ion relative to the unit cell crystalline axes and thus not sensitive to the ionic rotation [20]. There is a possible splitting of the mode near $500 \mathrm{~cm}^{-1}$ at the highest pressure studied (18.9 GPa).

The frequencies of selected modes are plotted with pressure in Fig. 3. A distinct change in slope is observed in the line commencing near $630 \mathrm{~cm}^{-1}$ near $11.7 \mathrm{GPa}$ as well as mild discontinuities in a few lines near 7.7 GPa, adding further support to our claim of a new phase transition near these pressures.

After pressurizing the sample to $18.9 \mathrm{GPa}$ (the highest pressure investigated), the sample was decompressed to near ambient conditions. The Raman spectrum of the decompressed sample indicates that the pressure-induced changes were completely reversible (see Fig. 4).

\section{Results (Pressure Dependence of X-ray Induced Decomposition):}

Figures $5 \mathrm{a}$ and $5 \mathrm{~b}$ display typical $\mathrm{x}$-ray diffraction patterns at two different sample irradiation locations and two different pressures for set irradiation times. Figure $5 c$ displays the sample as viewed with a microscope (through one diamond) after portions of the sample were irradiated. In Figure 5a, the sample was pressurized to 2.0 GPa and irradiated for 30 minutes. As is apparent from the diffraction pattern, the irradiated sample region has already largely and rapidly decomposed into $\mathrm{KCl}$ and $\mathrm{O}_{2}$ via the reaction: $\mathrm{KClO}_{4} \stackrel{h v}{\longrightarrow} \mathrm{KCl}+2 \mathrm{O}_{2}$, and $\mathrm{KCl}$ diffraction lines are present within seconds. Oxygen lines are not present due to the relatively low scattering power of oxygen and the fact that oxygen is a liquid at this pressure [23-24]. 
In Figure 5b, the same sample was further pressurized to $7.2 \mathrm{GPa}$ and translated relative to the $\mathrm{x}$-ray beam so that a virgin/fresh region of the sample was irradiated for another 30 minutes. Solid oxygen diffraction lines were observed (along with $\mathrm{KCl}$ diffraction lines) and are highlighted. When translating the sample to a new region for irradiation (relative to the fixed $\mathrm{x}$-ray beam), we ensured that no $\mathrm{KCl}$ or solid oxygen lines were initially present to prove that the irradiated region was indeed virgin.

In Figure 5c, the cross-like image in the sample hole represents the irradiation damage imprint caused by repetitive translation of the sample to find the sample center each time the pressure was increased. Damage spots represent regions that were irradiated at a given pressure. Each different spot represents a new pressure. We examined the growth of less mobile $\mathrm{KCl}$ (instead of $\mathrm{O}_{2}$ ) as molecular oxygen will likely diffuse throughout the sample [3] and thus would not serve as a reliable marker of decomposition progress.

The decomposition of $\mathrm{KClO}_{4}$ proceeded rapidly in the 16 ID-B beamline and the rate significantly slowed with pressure. The integrated diffraction spectra stacked as a function of time are plotted in Figure 6. In Fig 6a, only $\mathrm{KCl}$ is present in the $\mathrm{B}_{1}$ or rocksalt phase (Pm-3m, ICSD \#31232) [25] as the $\mathrm{KClO}_{4}$ rapidly decomposed in the focused $5 \mu \mathrm{m} \times 5 \mu \mathrm{m} x$-ray beam. In Fig. $6 \mathrm{~b}$, stacked diffraction patterns are shown as a function of time. New diffraction lines that appear are from $\beta-\mathrm{O}_{2}$ (Fmmm, ICSD \#37059) [24] and $\mathrm{KCl}$ in the $\mathrm{B}_{2}$ phase $(\mathrm{Pm}-3 \mathrm{~m})[7,25]$ and correlate with the predicted bars at the bottom of the Figure. Fig. 6c demonstrates that oxygen is now in the $\varepsilon$ phase (with $\mathrm{C} 2 / \mathrm{m}$ symmetry) [23-24], and $\mathrm{KCl}$ remains in the $\mathrm{B}_{2}$ phase $[7,25]$ at $15.2 \mathrm{GPa}$ as expected.

We present the results of our pressure-dependent decomposition study in Figure 7. It is evident that the growth of $\mathrm{KCl}$ slows with pressure. This slowing of the decomposition 
rate with pressure has been observed in other compounds (such as PETN and TATB [1516] and $\mathrm{KClO}_{3}$ [7]). Pressure in this case slows the decomposition reaction presumably because the sign of the volume change of the activated reaction complex is positive [15$16]$.

\section{Discussion:}

We successfully performed a Rietveld refinement at 7.2 GPa using the ambient orthorhombic symmetry (see Fig. 8). [We note in passing that a mixed phase $\left(\mathrm{O}_{2}\right.$ and $\left.\mathrm{KCl}\right)$ Rietveld refinement was unsuccessfully attempted for a longer 30 minute exposure time; also at 7.2 GPa. The results can be observed in the ancillary Fig. Sx.] Thus, as this suggests no fundamental structural change at 7.2 GPa, we do not suspect a structural phase transition commencing at 2.4 GPa but rather a "soft" polymorphic-type transition relating to varying orientations of the $\mathrm{ClO}_{4}{ }^{-}$anions within the unit cell which does not alter the fundamental unit cell symmetry. These polymorphic transitions are often sluggish [26] particular under the nonhydrostatic conditions we employed. No apparent phase transition was observed in the high pressure mixture of $\mathrm{KMnO}_{4}$ and $\mathrm{KClO}_{4}$ study up to 5.5 GPa [19]. At 7.7 GPa, the leftward shoulder on the $v_{1}$ line has now disappeared. Thus, we suspect that the first phase transition commences near 2.4 GPa but completes near 7.7 GPa. Another possibility is that no phase transition occurs at 2.4 GPa (notwithstanding the observation of a new peak) and the first pressure-induced phase transition occurs instead near 7.7 GPa. Further measurements to clarify this are planned.

When examining Fig. 6, it is evident that the x-ray induced decomposition of $\mathrm{KClO}_{4}$ occurred rapidly at $2 \mathrm{GPa}$, and that no diffraction pattern of $\mathrm{KClO}_{4}$ could be obtained be- 
cause it has already converted to $\mathrm{KCl}$ within the range of the $\mathrm{x}$-ray focused beam. At 7.2 and 15.2 GPa, however, the patterns of $\mathrm{KClO}_{4}$ are discernible at early times $(\mathrm{t}=0 \mathrm{~min}$.), and appear to differ considerably relative to each other, lending credence to a phase transition above 7.2 GPa. Thus, for some materials that decay rapidly in the presence of hard x-rays, pressure can be used to slow x-ray photochemical decomposition and enable longer time acquisitions and thus improved quality x-ray diffraction patterns.

In this experiment, we also present for the first time evidence for the growth of molecular oxygen in the crystalline form via x-ray diffraction. The observation can be explained as following: During irradiation, oxygen is likely produced as radicals that ultimately form interstitial molecular oxygen which then diffuse throughout the damaged and undamaged regions of the sample [3]. Over time, the oxygen molecules appear to coalesce with one another and form bulk polycrystalline oxygen at pressures above 2 GPa. One major goal of our x-ray experiment entailed verifying this explanation. Thus, we present for the first time, x-ray diffraction evidence demonstrating the crystallization of solid molecular oxygen that has been produced following extensive irradiation of $\mathrm{KClO}_{4}$ at extreme conditions. This significant observation implies that oxygen has chemically segregated. Observing the XRD pattern displaying oxygen lines in Fig. 5, it is apparent that the crystalline oxygen is in a good quality powder form with continuous diffraction rings. The lack of observation of crystalline oxygen at $2 \mathrm{GPa}$ pertains to the fact that the oxygen molecules are in a liquid state at this pressure [23]. 


\section{Conclusion:}

We have performed two studies of potassium perchlorate, an important inorganic oxidizer at high pressure to examine the compound's behavior under extreme conditions in an effort to aid useful hard x-ray photochemistry. In the first study, which is the first high pressure Raman study of this compound to the best of our knowledge, we observed at least two phase transitions via Raman spectroscopy: The first is likely sluggish between 2.4 and 7.7 GPa and does not appear to involve a physical alteration of molecular units but rather is polymorphic in nature associated with rotations or distortions of the $\mathrm{ClO}_{4}{ }^{-}$anion. The second phase transition near $11.7 \mathrm{GPa}$ has a structural change associated with it based on x-ray diffraction evidence from the second experiment. Upon sample decompression to near ambient pressure, the sample appeared to be unchanged, implying that all phase transitions were reversible upon decompression.

In the pressure-dependent x-ray photochemical decomposition study, a steady decrease in the decomposition rate was observed with increase in pressure which has been observed in other systems [15-16], including in $\mathrm{KClO}_{3}$ [7]. Due to rapid ionization from the powerful synchrotron x-ray beam (order of seconds to minutes) and the fact that oxygen is a liquid around $2 \mathrm{GPa}$, determination of the $\mathrm{x}$-ray structure of $\mathrm{KClO}_{4}$ with pressure was not possible but may be possible at higher pressures where x-ray induced decomposition significantly slows. Thus, pressure can provide a means of slowing down decomposition of irradiated compounds in the spirit of enabling more time to study the compounds.

These results provide important benchmark data that will assist in harnessing the oxidative potential of this relatively and ubiquitously utilized simple inorganic salt to en- 
able novel chemistry under extreme conditions. We also have encouraging evidence that high pressure studies of purified/crystallized simple molecules (such as the sparsely investigated halogens) can be conducted using our methods.

\section{Acknowledgements:}

We thank Quinn Smith and Blake Harris for aid. We acknowledge support from the Department of Energy National Nuclear Security Administration under Award Number(s) DE-NA0000979. We also acknowledge support from the DOE Cooperative Agreement No. DE-FC08-01NV14049 with the University of Nevada, Las Vegas. Portions of this work were performed at HPCAT (Sector 16), Advanced Photon Source (APS), Argonne National Laboratory. HPCAT operations are supported by DOE-NNSA under Award No. DE-NA0001974 and DOE-BES under Award No. DE-FG0299ER45775, with partial instrumentation funding by NSF. APS is supported by DOEBES, under Contract No. DE-AC02-06CH11357. 


\section{References:}

1) T. Davis, The Chemistry of Powder and Explosives, Angriff Press, Las Vegas (1943).

2) M. Pravica, D. Popov, S. Sinogeikin, D. Sneed, G. Guardala, Q. Smith, Appl. Phys. Lett. 103, 224103 (2013).

3) M. Pravica, D. Sneed, M. White and Y. Wang, J. Chem. Phys. 141, 091101 (2014); http://dx.doi.org/10.1063/1.4894402.

4) M. Pravica, M White, Y. Wang, APS SCCM 2015 Conf. Proc., accepted for publication (2015).

5) M. Pravica, L. Bai, C. Park, Y. Liu, M. Galley, J. Robinson, N. Bhattacharya, Rev. Sci. Inst. 82, 106102 (2011).

6) M. Pravica et al., Rev. Sci. Inst. 83, 036102 (2012).

7) M. Pravica, L. Bai, N. Bhattacharya, J. Appl. Cryst. 45, pp. 48-52 (2012).

8) M. Pravica, D. Sneed, L. Bai, C. Park, J. Phys. Chem. 117, pp. 2302-2306 (2013).

9) M. Pravica, B. Hulsey, L. Bai, and D. Sneed, Q. Smith and G. Guardala, 18th APS-SCCM \& 24th AIRAPT Conf. Proc. J. Phys: Conf. Series 500, 022009 (2014).

10) M. Pravica, Y. Liu, L. Bai, Chem. Phys. Lett. 555, pp. 113-115 (2013).

11) M. Pravica, D. Sneed, Q. Smith, L. Bai, Chem. Phys. Lett. 530, pp. $74-76$ (2013).

12) M. Pravica, D. Sneed, Y. Wang, Q. Smith and G. Subrahmanyam, J.Chem. Phys. 140, 194503 (2014).

13) M. Pravica, D. Sneed, M White, Y. Wang, Rev. Sci. Instr. 85, 086110 (2014). 
14) N. Yedukondalu, V. Ghule, and G. Vaitheeswaran, J. Chem. Phys., 138, 174701 (2013).

15) H. Giefers, M. Pravica, P. Liermann, W. Yang, Chem. Phys. Lett. 429, pp. 304309 (2006).

16) H. Giefers, M. Pravica, J. Phys. Chem. A 112 (15), pp. 3352-3359 (2008).

17) C. Pistorius, J. Phys. Chem. Solid. 31, pp. 385-389 (1970).

18) T. Tatsuki, S. Adachi, M. Itoh, T. Tamura, X. Wu, C. Jin, N. Koshizuka, K. Tan Physica C 255, pp. 61-64 (1995).

19) M. Barsan, I. Butler, Denis Gilson, J. Phys. Chem. B, 110, pp. 9291-9297 (2006).

20) N. Toupry, H. Poulet, M. Le Apostolic, R. Pick, M. Yvinec, J. Raman Spect. 14, pp. 166-177 (1983).

21) G. Johannsen, O. Lindqvist, Acta Cryst. B, 33, pp. 2918-2919 (1977).

22) http://www.space.com/1099-space-station-crew-ignite-oxygen-generatingcandles.html

23) F. Gorelli, M. Santoro, L. Ulivi, M. Hanfland, Phys. Rev. B, 65, 172106 1-4 (2002).

24) M. Nicol, K.R. Hirsch, H.B. Holzapfel, Chem. Phys. Lett. 68, pp. 49-52 (1979).

25) http://cheiron2010.spring8.or.jp/text/bl/4_BL04B1.pdf

26) M. Pravica, YR Shen+, Z. Quine, E. Romano and D. Hartnett, J. Phys. Chem. B, 111, pp. 4103-4108 (2007). 


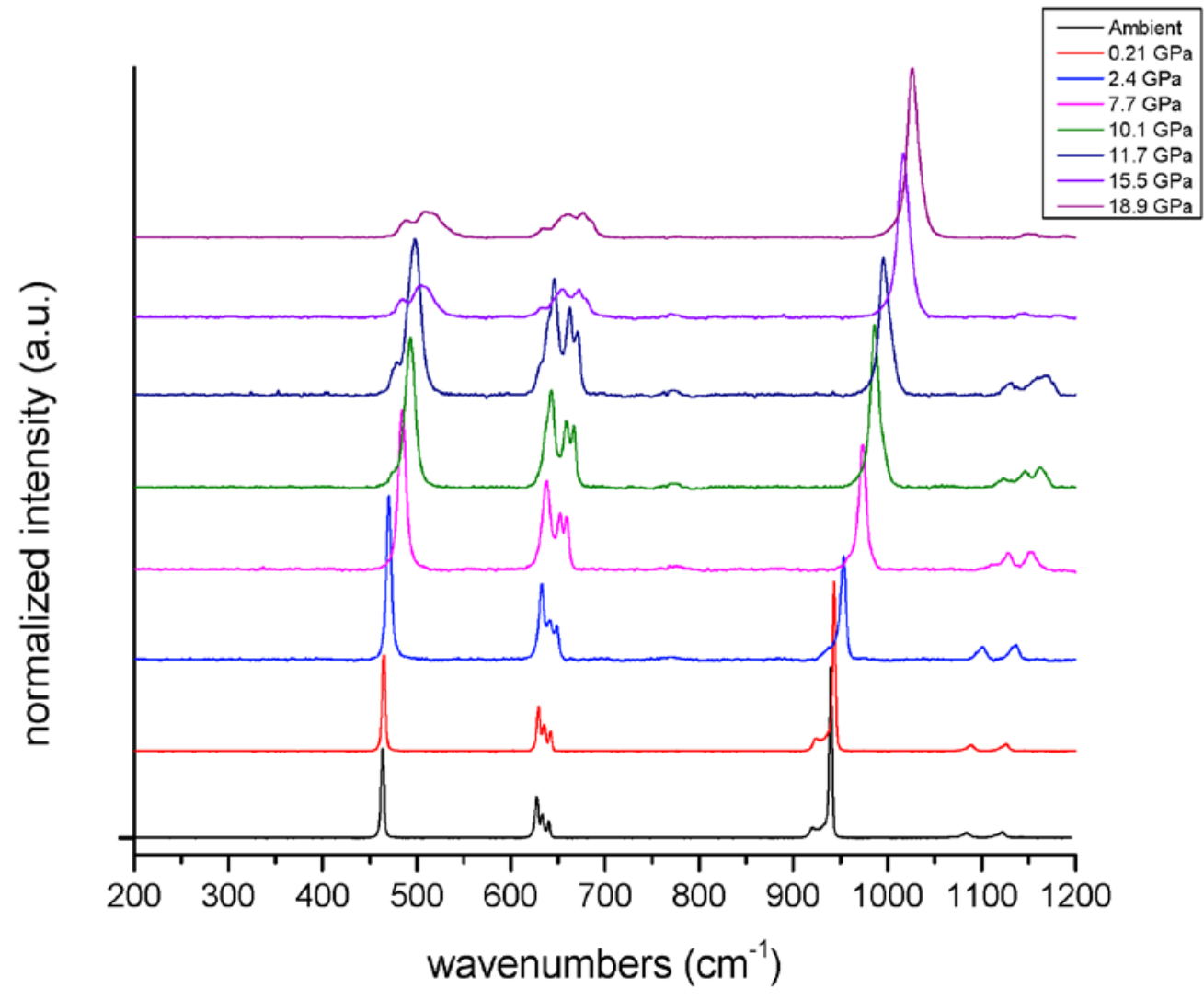

Figure 1: Raman spectra of $\mathrm{KClO}_{4}$ as a function of pressure in the low wavenumber region (200-1200 $\left.\mathrm{cm}^{-1}\right)$. New peaks emerge in the 2.4. GPa and 7.7 GPa plots suggest a sluggish phase transition that commences near 2.4 GPa and ends around 7.7 GPa. Above 11.7 GPa, the some (not all) of the Raman spectral modes are considerably broadened. 


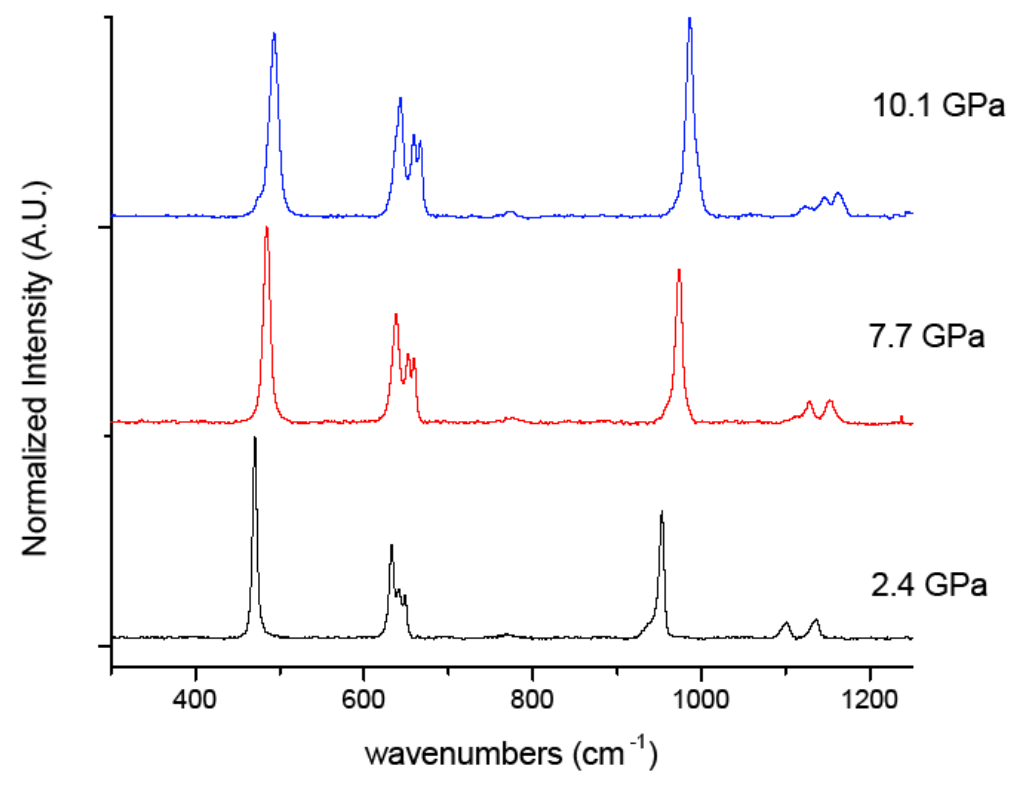

Figure 2: Selected Raman plots from Fig 1 better illustrating the new peak that appears near $1120 \mathrm{~cm}^{-1}$ in the $10.1 \mathrm{GPa}$ plot. 


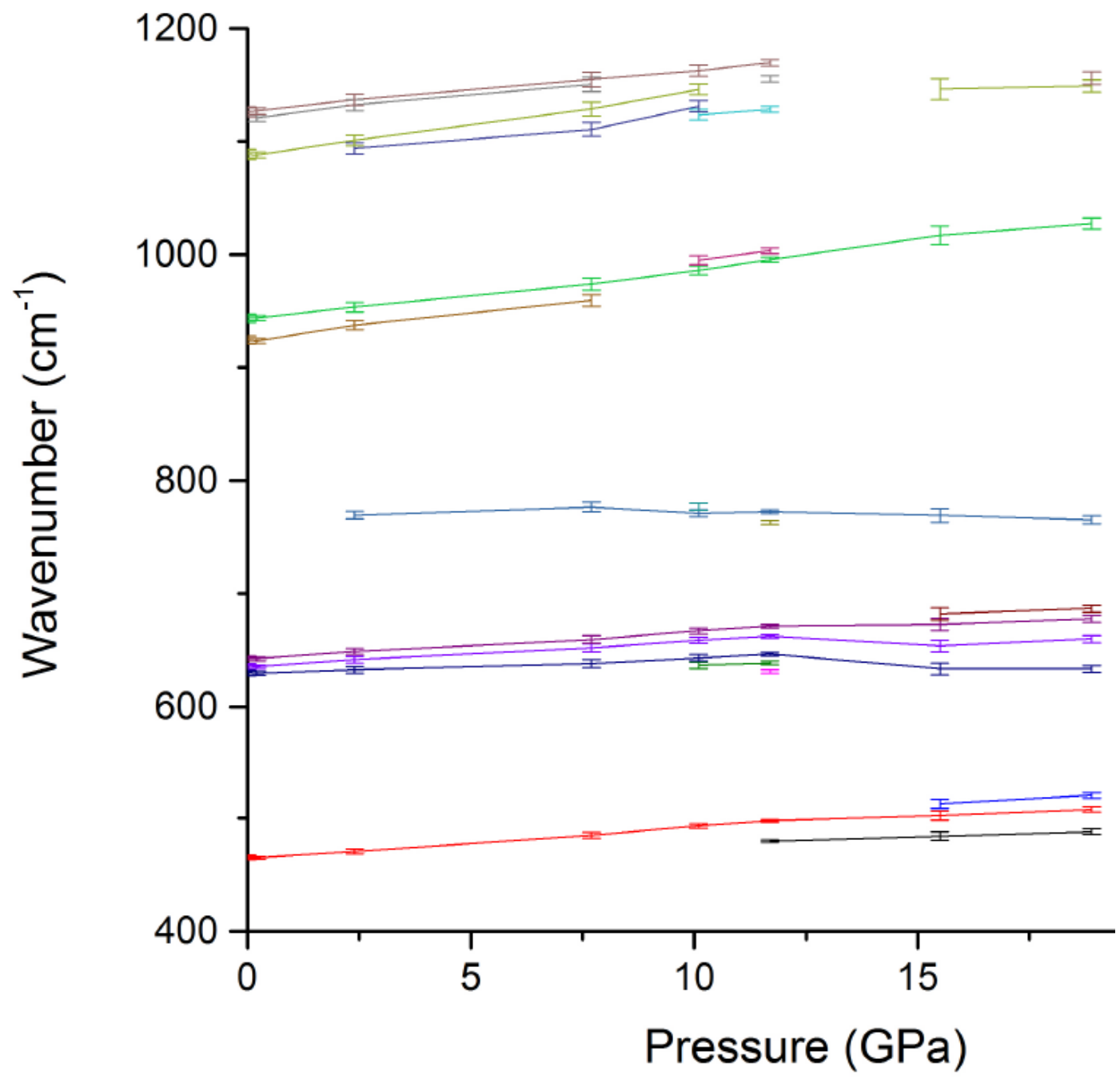

Fig 3: Raman spectral plots of various modes plotted against pressure. 


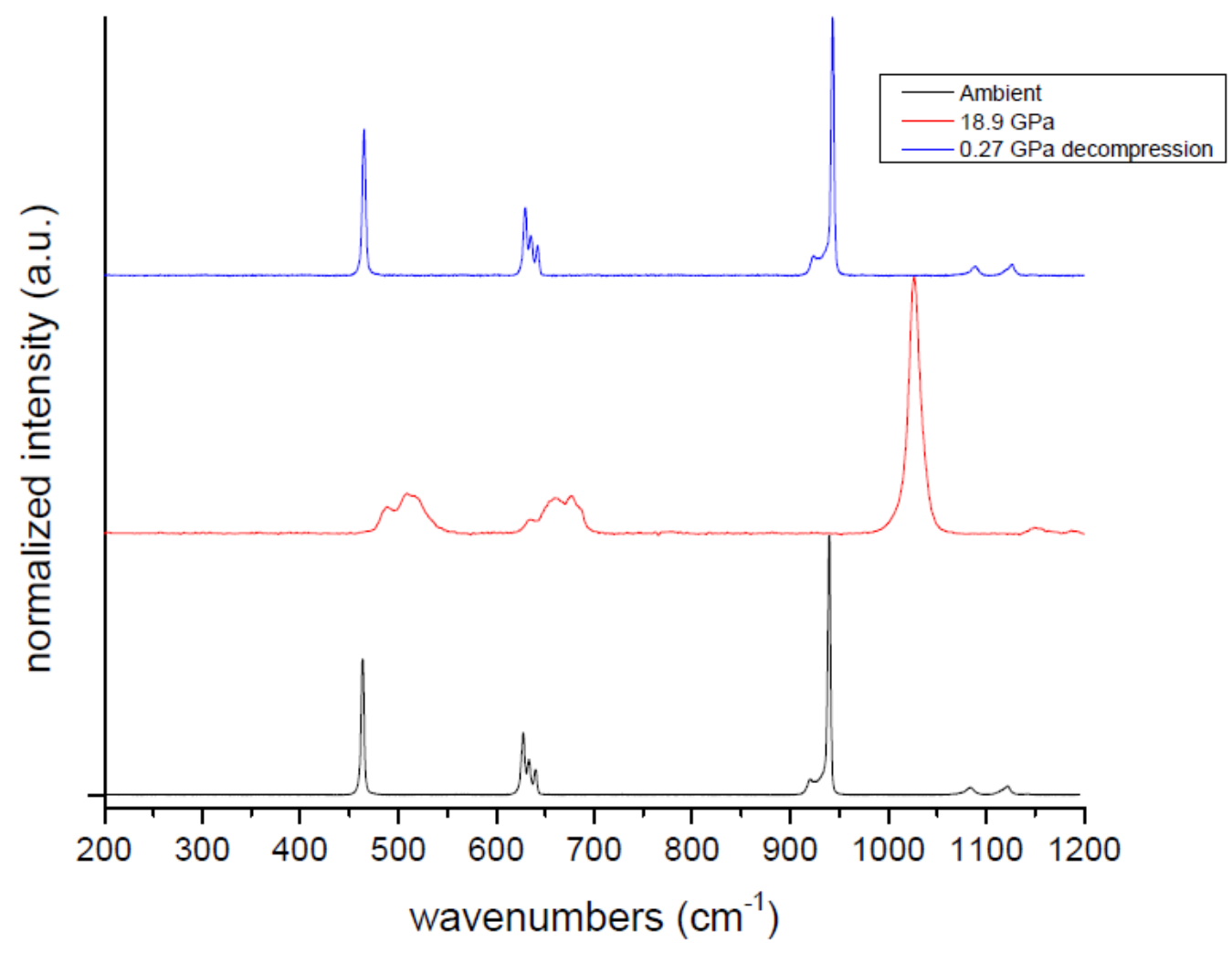

Fig. 4: Pressure cycling of the $\mathrm{KClO}_{4}$ sample suggests that pressure-induced effects are completely reversible. 

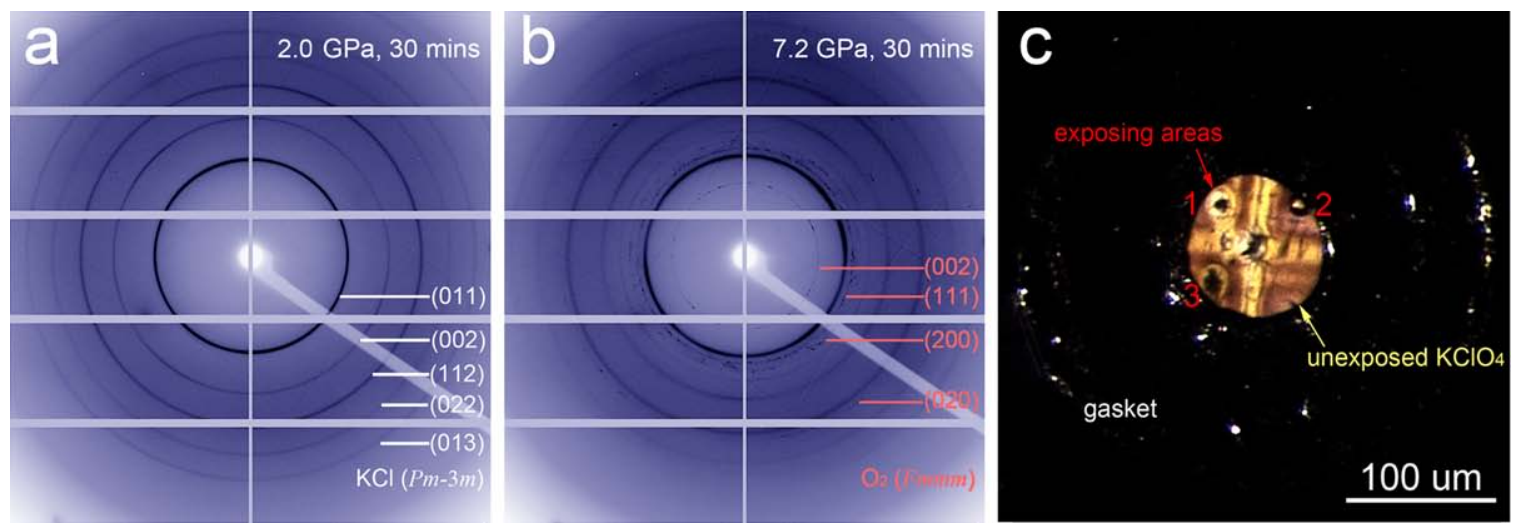

Figure 5: a) Diffraction pattern of $\mathrm{KClO}_{4}$ pressurized to $2.0 \mathrm{GPa}$ and irradiated for 30 minutes. The pattern already shows a preponderance of $\mathrm{KCl}$ diffraction lines and much weaker $\mathrm{O}_{2}$ lines; b) $\beta-\mathrm{O}_{2}$ diffraction lines observed in irradiated $\mathrm{KClO}_{4}$ irradiated for 30 minutes at a pressure of 7.2 GPa; c) Photograph showing irradiated versus unirradiated regions of a $\mathrm{KClO}_{4}$ sample. The cross represents damage created when the sample is translated and briefly irradiated during sample alignment in the synchrotron x-ray beam. 

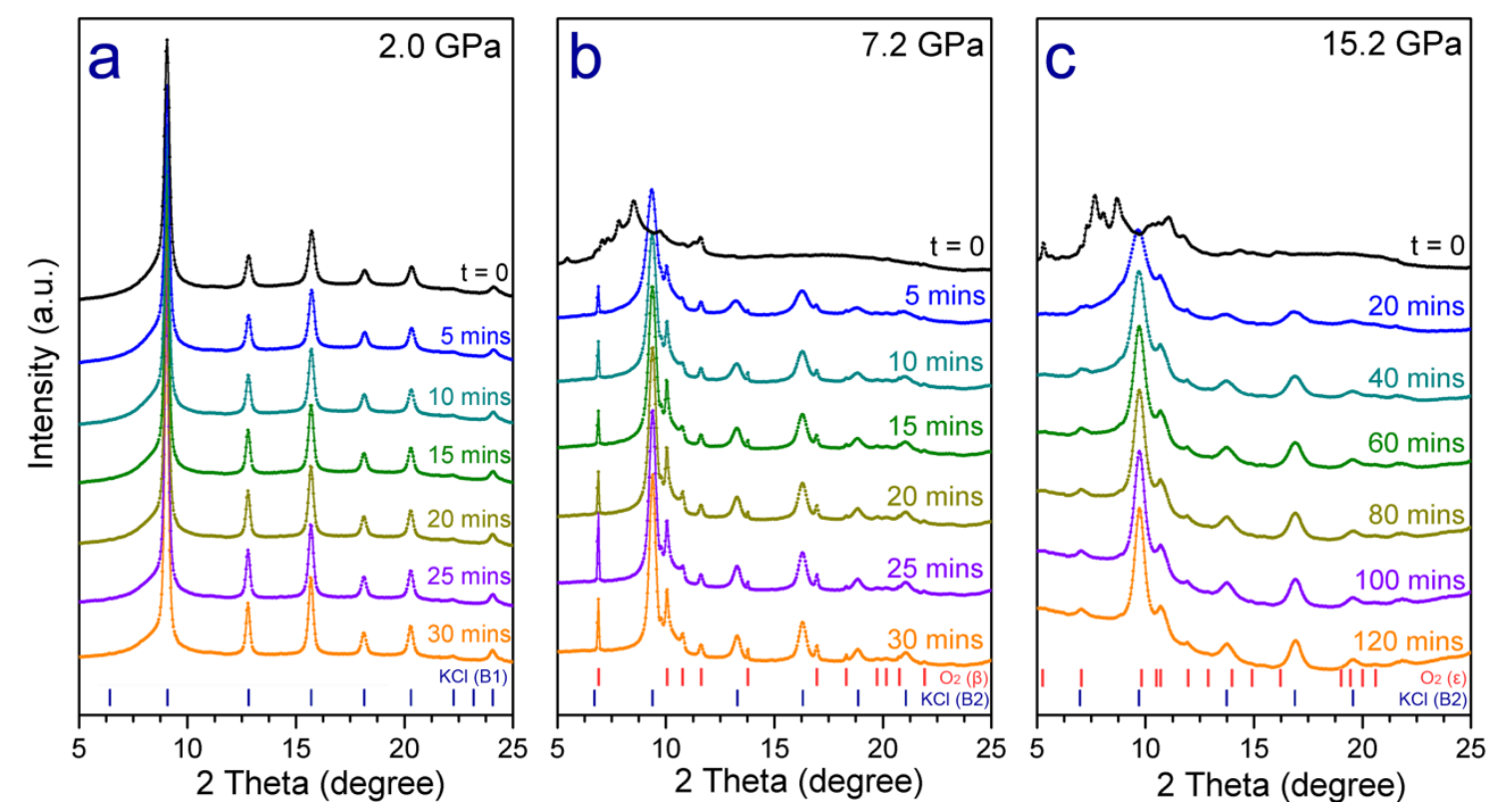

Figure 6 (a,b,c): Powder synchrotron X-ray diffraction patterns $(\lambda=0.406626 \AA)$ of $\mathrm{KClO}_{4}$ as a function of exposure time under different pressure: (a) $2.0 \mathrm{GPa}$, (b) $7.2 \mathrm{GPa}$ and (c) $15.2 \mathrm{GPa}$. The lower short vertical bars on the bottom of the plots represent the main theoretical Bragg reflection positions of $\mathrm{KCl}$ in the $\mathrm{B}_{1}$ phase (a) and $\mathrm{B}_{2}$ phase (b and c). The higher bars represent predicted crystalline $\mathrm{O}_{2}$ diffraction lines in the $\beta$ phase (b) and $\varepsilon$ phase (c) and $\mathrm{O}_{2}$. 


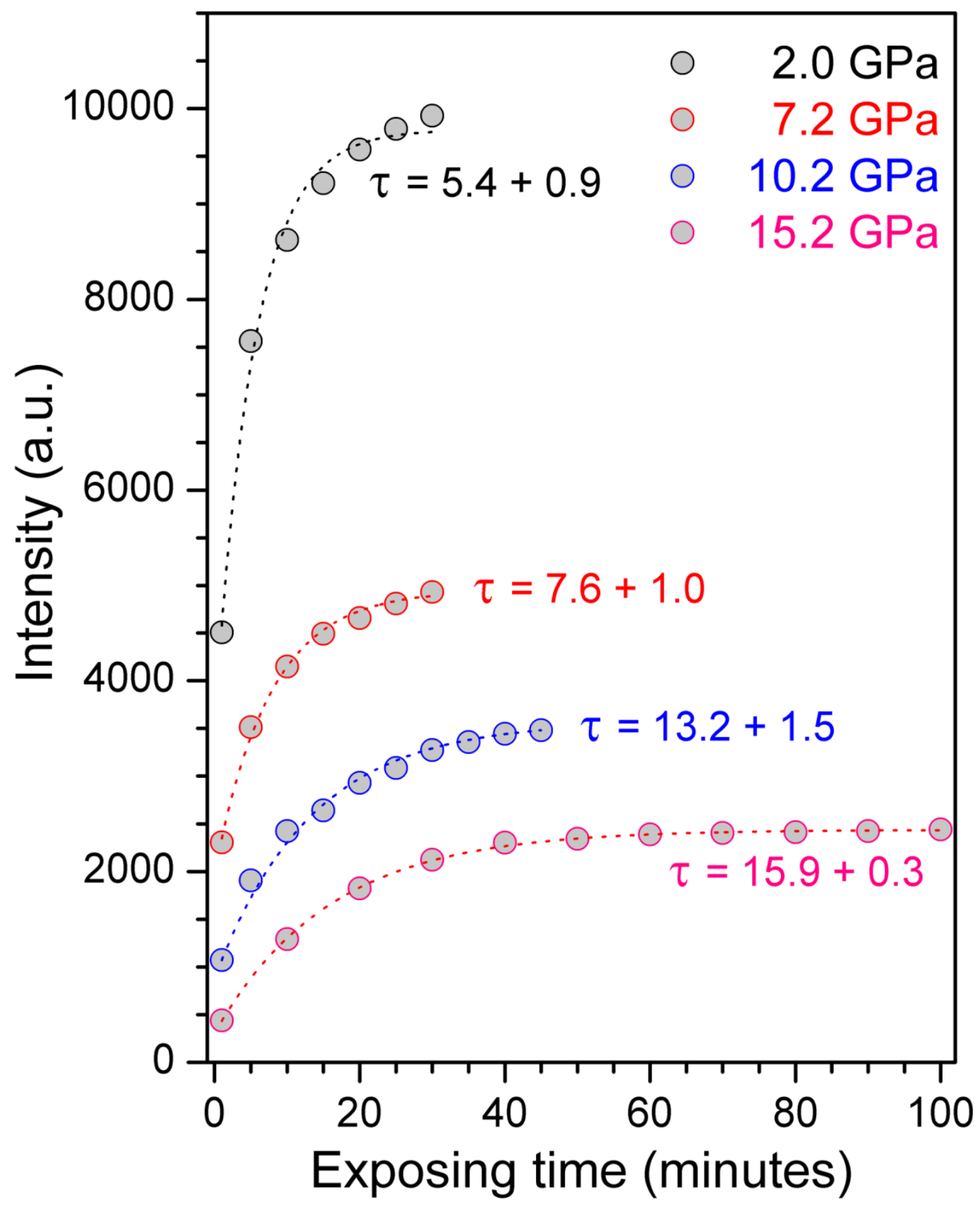

Figure 7: The pressure dependence of the growth of the (011) diffraction peak of KCl (B2 structure) as a function of exposure time. The growth constant $\tau$ (in minutes) from the $\left(1-e^{-t / \tau}\right)$ fitting function is included for each curve. 


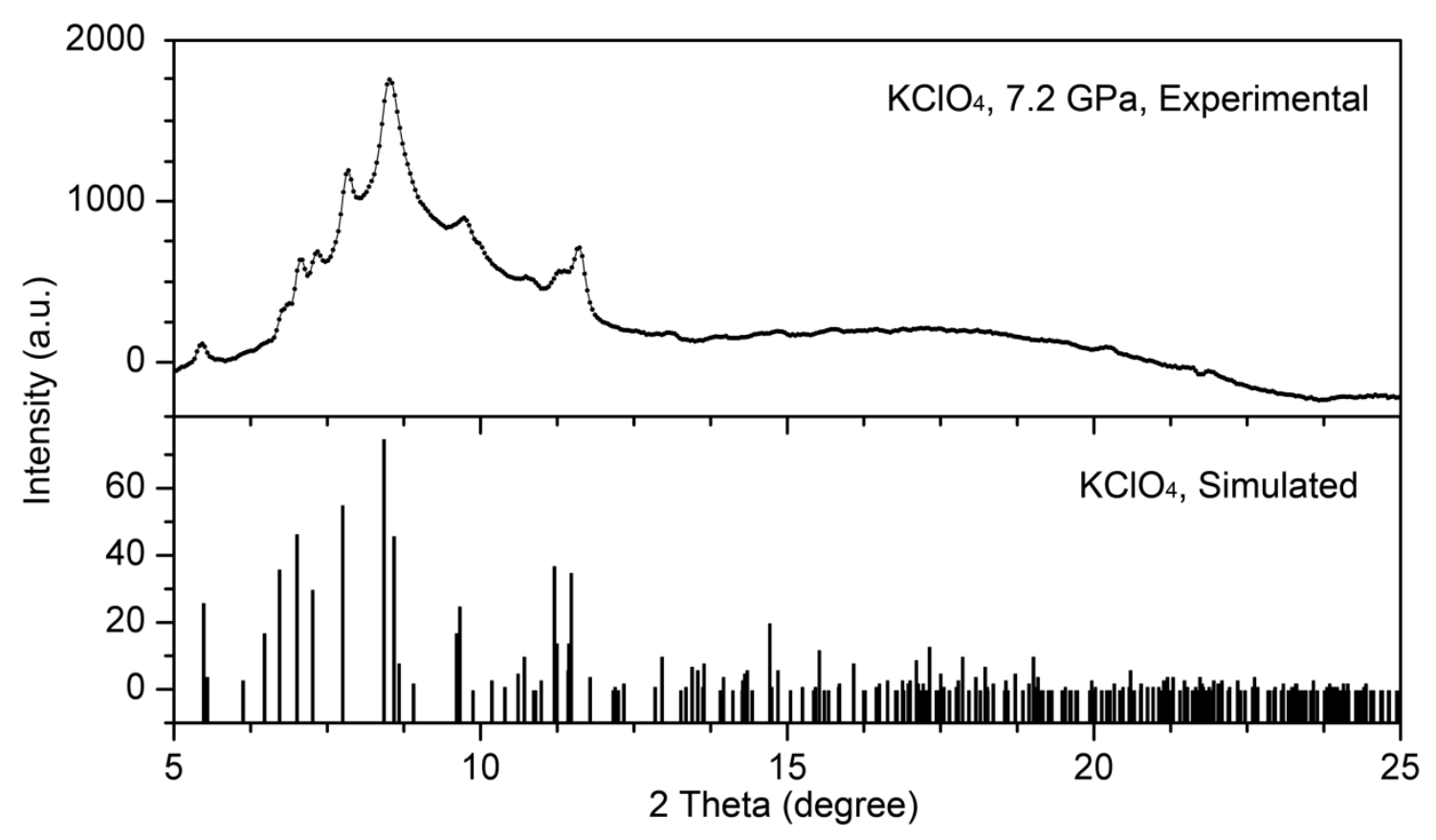

Figure 8: Rietveld refinement of the $\mathrm{KClO}_{4}$ sample using ambient orthorhombic symmetry ( $\mathrm{t}=0$ sec pattern). 
Graphical Abstract

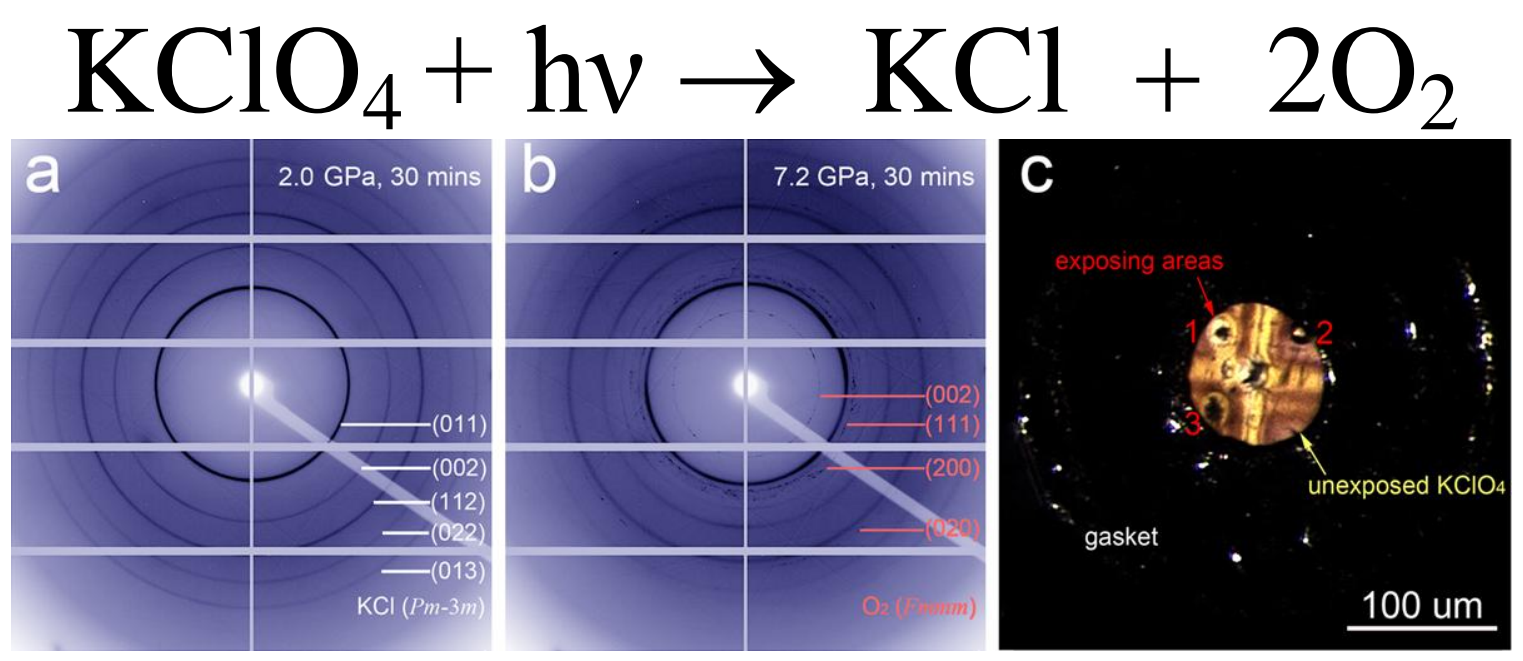

